\title{
Adsorção de nitrato em solos com cargas variáveis
}

\author{
Marco Aurélio Kondracki de Alcântara(1) e Otávio Antonio de Camargo(2)
}

\begin{abstract}
(1)Faculdade de Engenharia Química de Lorena, Dep de Biotecnologia, Caixa Postal 116, Rod. Itajubá-Lorena, Km 74,5, Bairro Campinho, CEP 12600-970 Lorena, SP. E-mail: maka@debiq.faenquil.br (2)Instituto Agronômico, Centro de Pesquisa e Desenvolvimento de Solos e Recursos Ambientais, Caixa Postal 28, CEP 13020-970 Campinas, SP. E-mail: ocamargo@iac.sp.gov.br
\end{abstract}

Resumo - O objetivo deste trabalho foi avaliar o comportamento adsortivo do nitrato em relação às modificações da carga líquida do solo decorrentes da adição de ácidos, bases (carbonatos), fosfato e sulfato. Foram utilizadas amostras dos horizontes A e B de dois solos: Latossolo Vermelho acriférrico e Nitossolo Vermelho eutrófico. Para verificar o comportamento adsortivo do nitrato em relação aos tratamentos, ajustaram-se isotermas de adsorção de Freundlich (exponencial). O modelo de Freundlich descreve satisfatoriamente a adsorção do nitrato ao solo em todos os tratamentos. Existe correlação significativa entre o pH e a carga líquida dos solos, considerando-se os tratamentos aplicados ao horizonte B do Latossolo Vermelho. A adsorção do nitrato nos horizontes A e B comporta-se de maneira diferenciada nos dois solos utilizados. No Latossolo Vermelho, a adsorção é maior no horizonte subsuperficial em relação ao superficial. No Nitossolo Vermelho, a adsorção é maior no horizonte superficial em relação ao subsuperficial. As adições de sulfato e de fosfato ao solo resultam em pequena diminuição na adsorção de nitrato em relação ao observado no horizonte B do Latossolo Vermelho acriférrico.

Termos para indexação: solos tropicais, nitrogênio, carga líquida.

\section{Nitrate adsorption in variable charged soils}

\begin{abstract}
The objective of this work was to assess the adsorptive behavior of nitrate due to net charge $(\mathrm{pH})$ modifications caused by the addition of acids, bases (carbonates), phosphate and sulfate. Samples of A and B horizons of two soils: an Ustox and an Ustalf . Freundlich isotherms were adjusted in order to verify the influence of the different treatments. The Freundlich model fits well to experimental data. There is significance correlation between $\mathrm{pH}$ and net charge for the soils, considering the treatments applied to the B horizon of the Ustox soil. Nitrate adsorption in the A and B horizons is different for the two soils. In Ustox soil, adsorption is greater in subsuperficial than in superficial horizon. In Ustalf soil, adsorption is greater in the superficial horizon than in the subsuperficial one. Sulfate and phosphate additions result in little reduction on the nitrate adsorption comparing to the Ustox B horizon.
\end{abstract}

Index terms: tropical soils, nitrogen, net charge.

\section{Introdução}

A adsorção do nitrato pelo solo tem relevância direta na sua dinâmica e, conseqüentemente, no manejo da adição desse ânion, seja por fertilização mineral ou aplicação de vários resíduos de origem agrícola ou industrial (Black \& Waring, 1976; Bowden et al., 1980; Dynia \& Camargo, 1999).

Do ponto de vista eletroquímico, existe uma grande diferença entre a adsorção de nitrato ao solo e a de outros oxiânions, pois ele não é adsorvido especificamente, sendo sua adsorção apenas eletrostática, dependente exclusivamente da carga do solo. Existem solos nos quais as cargas positivas ocorrem em quantidades apreciáveis em condições naturais de pH e de maneira significativa nos horizontes subsuperficiais. A presença de cargas positivas é muito maior em solos de regiões tropicais úmidas, com baixos teores de matéria orgânica e em estádio de intemperismo avançado do que em solos de regiões temperadas. Se as partículas do solo carregarem cargas positivas, a lixiviação do nitrato poderá ser dificultada, embora não totalmente impedida. No entanto, o nitrato não é o único ânion cuja lixiviação é influenciada pelo $\mathrm{pH}$ e pelas cargas elétricas do solo (Raij \& Camargo, 1974).

Camargo \& Raij (1989) detectaram que a movimentação de $\mathrm{SO}_{4}{ }^{2-}$ foi afetada pela carga líquida do solo, alterada pela modificação do $\mathrm{pH}$ em um Latossolo Ver- 
melho-Amarelo álico. Em um Latossolo, Black \& Waring (1976) detectaram adsorção de nitrato em profundidades maiores que $20 \mathrm{~cm}$. Esses pesquisadores encontraram correlação positiva entre a adsorção de nitrato e a carga elétrica líquida $\left(\Delta \mathrm{pH}=\mathrm{pH}_{\mathrm{KCl}}-\mathrm{pH}_{\mathrm{H} 2 \mathrm{O}}\right)$. A retenção de nitrato também é dependente da sua concentração e da presença de outros ânions no solo, sendo que doses crescentes de nitrato de amônio aumentaram a profundidade de lixiviação desse ânion. A adição de sulfato ao subsolo também favoreceu a movimentação de nitrato (Black \& Waring, 1976).

A preferência dos sítios de adsorção pelos íons $\mathrm{NO}_{3}{ }^{-}$, $\mathrm{SO}_{4}{ }^{2-}$ e $\mathrm{H}_{2} \mathrm{PO}_{4}{ }^{2-}$ foi estudada no México em subsolos de Latossolos, Argissolos e Andisols (este último, sem correspondência no Sistema Brasileiro de Classificação), utilizando-se soluções contendo diferentes combinações e concentrações desses ânions. Tanto a adição de fosfatos como a de sulfatos provocou aumento das cargas negativas do solo, ocasionado pela sua própria adsorção, o que favoreceria ainda mais a lixiviação do nitrato, uma vez que o mesmo é adsorvido eletrostaticamente. Em experimentos de movimentação de íons em colunas de solo acondicionado utilizando um Argissolo distrófico, Bellini et al. (1996) avaliaram o efeito da diminuição da capacidade de troca de ânions (CTA) no seu movimento e constataram que o aumento do $\mathrm{pH}$ do solo obtido com a calagem resultou em diminuição da CTA, aumento da capacidade de troca de cátions (CTC) e, conseqüentemente, diminuição algébrica da carga líquida, que ficou mais negativa. Para predizer a movimentação de um elemento nesse tipo de solo, é necessário estimar a influência da carga do subsolo no seu retardamento. Em um Latossolo Vermelho-Escuro do Cerrado, Dynia \& Camargo (1999) detectaram aumento na capacidade de retenção de nitrato (CRN) com a profundidade. Na camada superficial $(0-20 \mathrm{~cm})$, a CRN foi negativa (indicando exclusão de ânions) e na subsuperficial $(20-60 \mathrm{~cm})$, apresentou valores positivos. Na camada subsuperficial, tanto a adição de fosfatos como a calagem reduziram a capacidade de retenção de nitrato, sendo que o efeito da calagem foi muito mais pronunciado que o da adição de fosfatos.

A manipulação da carga pela influência do pH e da presença de carbonatos, fosfatos e sulfatos na adsorção de nitrato é importante em termos práticos. A incorporação de calcário e de fosfatos ao solo aumenta a quantidade de cargas negativas do solo, o que pode favorecer a lixiviação de ânions (Naidu et al., 1990). Em experimentos realizados no campo em um
Argissolo distrófico da Nigéria, Wong et al. (1992) avaliaram a influência do cultivo de plantas conjuntamente com a calagem, adição de Mg, K e de uréia na lixiviação de nitrato e de outros íons no solo, utilizando lisímetros para monitorar as perdas por percolação. Nas parcelas não cultivadas, a adição de uréia aumentou a lixiviação de nitrato e de outros íons. Porém, o efeito da adição de fertilizantes e corretivos nas perdas de nitrato foi aproximadamente quatro vezes maior nas parcelas cultivadas com milho e arroz. Tais resultados revelam que experimentos envolvendo apenas aspectos físicos e químicos do solo, apesar de facilitarem o entendimento de alguns processos envolvidos, devem ser vistos tão somente como uma etapa no entendimento da dinâmica desse íon no solo.

O objetivo deste trabalho foi estudar o comportamento adsortivo do nitrato em relação às modificações da carga líquida do solo, decorrentes da adição de ácidos, bases (carbonatos), fosfato e sulfato.

\section{Material e Métodos}

Foram utilizadas amostras dos horizontes A e B de um Latossolo Vermelho acriférrico (LV) e de um Nitossolo Vermelho eutrófico (NV) (Embrapa, 1999), coletadas no Município de Ribeirão Preto, SP. As amostras foram coletadas nas profundidades de 0-20 cm e de 50-70 cm (aproximadamente $50 \mathrm{~kg}$ de cada horizonte), secadas ao ar, tamisadas em peneiras de malha de $2 \mathrm{~mm}$ e cuidadosamente homogeneizadas. A seguir, foram umedecidas novamente e armazenadas com umidade próxima a 0,03 MPa. Os atributos químicos e físicos das amostras utilizadas foram determinados de acordo com Camargo et al. (1986) (Tabela 1).

O método usado na determinação das cargas elétricas dos solos foi adaptado de Camargo et al. (1986), titulando-se amostras de solo com $\mathrm{OH}^{-}$e $\mathrm{H}^{+}$a três concentrações de eletrólitos. $\mathrm{O}$ solo foi previamente lixiviado com $\mathrm{HCl} 100 \mathrm{mmol} \mathrm{L}^{-1}$ e, em seguida, percolado com água destilada para remover o excesso de cloreto. As curvas de titulação foram obtidas com soluções contendo diferentes concentrações de $\mathrm{NaOH}(2,0$, 4,0, 8,0 e $\left.16,0 \mathrm{mmol} \mathrm{L}^{-1}\right)$ ou $\mathrm{HCl}\left(2,0,4,0\right.$ e $\left.6,0 \mathrm{mmol} \mathrm{L}^{-1}\right)$. Cada concentração de ácido ou base foi preparada tendo como eletrólito suporte $\mathrm{KCl}$ nas concentrações de $100,0,10,0$ e $1,0 \mathrm{mmol} \mathrm{L}^{-1}$. O ponto de efeito salino nulo (PESN) foi considerado como sendo o $\mathrm{pH}$ no qual ocorre a intersecção das retas ajustadas para as diferentes concentrações de KCl. No NV-A, NV-B, LV-A e LV-B, 
esses valores de pH corresponderam a 3,56, 3,22, 3,24 e 4,94, respectivamente. Na determinação da carga líquida (CL), foi feito um segundo gráfico, no qual se fez coincidir o PESN encontrado no primeiro gráfico com a carga zero na abscissa. Utilizando-se a mesma escala do gráfico anterior, colocaram-se de um lado do zero as cargas positivas e de outro as positivas, plotando-se contra o pH em equilíbrio. O valor da CL pôde ser encontrado na abscissa para um determinado $\mathrm{pH}$ e concentração do eletrólito de suporte de $\mathrm{KCl}$.

O estudo da adsorção de nitrato foi realizado conforme Selim \& Amacher (1997). As concentrações iniciais $\left(\mathrm{C}_{0}\right)$ utilizadas foram preparadas de modo a fornecer $\mathrm{NO}_{3}{ }^{-}$nas concentrações de $0,00,0,18,0,36,0,72$, 1,44, 2,16, 2,88, 4,32, 5,76 e 8,64 mmol L-1 de solução, utilizando-se como fonte o nitrato de potássio. Todas essas soluções foram preparadas tendo como suporte uma solução de $\mathrm{CaCl}_{2} 50$ mmol L-1. Colocaram-se $4 \mathrm{~g}$ de TFSA em tubos de centrífuga de plástico com tampa e, em cada tubo, adicionaram-se $20 \mathrm{~mL}$ de solução com uma concentração inicial de nitrato. Utilizaram-se duas repetições por $\mathrm{C}_{0}$ (vinte tubos para cada tratamento). A relação solo:solução (1:5) foi definida após ensaios preliminares. Agitaram-se os tubos durante 22 horas, a 150 osc min $^{-1}$ e, após centrifugação a $2.000 \mathrm{~g}$, durante 5 minutos, determinou-se o nitrato no sobrenadante em espectrofotômetro a $470 \mathrm{~nm}$, utilizando-se o método da brucina (Baker, 1969).

Foram feitos gráficos das concentrações adsorvidas em função das concentrações em equilíbrio $\left(\mathrm{C}_{\mathrm{e}}\right)$. Ajus-

Tabela 1. Caracterização química e física dos $\operatorname{solos}^{(1)}$.

\begin{tabular}{|c|c|c|c|c|}
\hline \multirow[t]{2}{*}{ Atributo } & \multicolumn{4}{|c|}{ Solo e horizonte } \\
\hline & NV-A & NV-B & LV-A & LV-B \\
\hline $\mathrm{pH} \mathrm{CaCl} 2$ & 5,3 & 5,5 & 4,6 & 5,0 \\
\hline P resina $\left(\mathrm{mg} \mathrm{dm}^{-3}\right)$ & 9,0 & 5,0 & 15,0 & 3,0 \\
\hline $\operatorname{MO}\left(\mathrm{g} \mathrm{dm}^{-3}\right)^{(2)}$ & 46,0 & 12,0 & 29,0 & 10,0 \\
\hline $\mathrm{Al}\left(\mathrm{mmol}_{\mathrm{c}} \mathrm{dm}^{-3}\right)$ & 0,0 & 0,0 & 4,0 & 0,0 \\
\hline $\mathrm{H}+\mathrm{Al}\left(\mathrm{mmol}_{\mathrm{c}} \mathrm{dm}^{-3}\right)$ & 42,0 & 26,0 & 52,0 & 28,0 \\
\hline $\mathrm{SB}\left(\mathrm{mmol}_{\mathrm{c}} \mathrm{dm}^{-3}\right)^{(3)}$ & 95,3 & 30,7 & 14,5 & 5,3 \\
\hline $\mathrm{CTC}\left(\mathrm{mmol}_{\mathrm{c}} \mathrm{dm}^{-3}\right)$ & 137,3 & 56,7 & 66,5 & 33,3 \\
\hline $\mathrm{CTC}_{\mathrm{pH}}\left(\mathrm{mmol}_{\mathrm{c}} \mathrm{dm}^{-3}\right)^{(4)}$ & 104,3 & 36,4 & 22,5 & 11,0 \\
\hline $\mathrm{CTA}_{\mathrm{pH}}\left(\mathrm{mmol}_{\mathrm{c}} \mathrm{dm}^{-3}\right)^{(4)}$ & 5,1 & 4,8 & 3,1 & 7,8 \\
\hline Fe oxalato $\left(\mathrm{g} \mathrm{kg}^{-1}\right)^{(5)}$ & 14,2 & 8,5 & 4,9 & 1,8 \\
\hline $\mathrm{Al}$ oxalato $\left(\mathrm{g} \mathrm{kg}^{-1}\right)^{(5)}$ & 2,6 & 3,4 & 2,7 & 3,0 \\
\hline Mn oxalato $\left(\mathrm{g} \mathrm{kg}^{-1}\right)^{(5)}$ & 0,7 & 0,7 & 0,7 & 0,6 \\
\hline Argila $\left(\mathrm{g} \mathrm{kg}^{-1}\right)$ & 537,0 & 651,0 & 587,0 & 596,0 \\
\hline Umidade a $300 \mathrm{kPa}^{(6)}$ & 0,546 & 0,269 & 0,269 & 0,368 \\
\hline
\end{tabular}

(1)NV: Nitossolo Vermelho eutrófico; LV: Latossolo Vermelho acriférrico. ${ }^{(2)} \mathrm{MO}$ : matéria orgânica. ${ }^{(3)} \mathrm{SB}$ : soma de bases. ${ }^{(4)} \mathrm{CTC}_{\mathrm{pH}}$ e $\mathrm{CTA}_{\mathrm{pH}}$ : capacidade de troca de cátions e de ânions a $\mathrm{pH}$ do solo, respectivamente. ${ }^{(5)} \mathrm{Fe}, \mathrm{Al}$ ou $\mathrm{Mn}$ oxalato: extraídos com oxalato de amônio. ${ }^{(6)}$ Determinada em câmara de pressão. taram-se isotermas de adsorção de Freundlich com o auxílio do programa computacional Microcal Origin v. 5.0, de acordo com a expressão: $\mathrm{S}=\mathrm{KC}_{\mathrm{e}}{ }^{\mathrm{n}}$, em que $\mathrm{C}_{\mathrm{e}}$ é a concentração do soluto em equilíbrio na solução ( $\left.\mathrm{kg} \mathrm{m}^{-3}\right)$; K é o coeficiente de adsorção (mol $\left.{ }^{(1-n)} \mathrm{m}^{3(\mathrm{n}-1)}\right)$; $n$ é uma estimativa do parâmetro de ajuste (adimensional); e $S$ é a massa do soluto associada à fase sólida, ou quantidade adsorvida ao solo $\left(\mathrm{kg} \mathrm{kg}^{-1}\right)$. O S foi calculado pela expressão $\left(\mathrm{C}_{0}-\mathrm{C}_{\mathrm{e}}\right) \mathrm{Fd}$. A variável Fd é o fator de diluição, considerando a relação solução/solo, no caso, 20/4.

Foram realizados três experimentos de adsorção de nitrato. No experimento I, estudou-se a eluviação do nitrato utilizando-se os horizontes A e B dos dois solos. Neste trabalho, embora tenha se convencionado denominar esses solos como em "condições naturais", é importante lembrar que eles foram previamente preparados, passando por alterações de natureza física e química, em relação às condições encontradas originalmente no campo. Os tratamentos utilizados neste experimento foram denominados de NV-A, NV-B, LV-A e LV-B, indicando a sigla e o horizonte de cada solo.

No experimento II, avaliou-se o efeito da alteração do pH (CL) e de carbonatos na adsorção de nitrato. Os tratamentos desse experimento foram aplicados ao horizonte B do LV (LV-B). As doses de corretivo (HCl ou $\mathrm{MgCO}_{3}$ ) possibilitaram a obtenção de níveis de $\mathrm{pH}$ abaixo e acima do PESN do solo, resultando em cargas líquidas positivas e negativas, respectivamente. Para diminuir o pH, procedeu-se a lixiviação do solo com soluções ácidas. Nesse estudo, foram utilizadas colunas de percolação de vidro de $4,5 \mathrm{~cm}$ de diâmetro interno e $40 \mathrm{~cm}$ de altura. Na base e no topo de cada coluna foram colocados discos de manta geotêxtil (bidim), para evitar a perda e o selamento superficial do solo durante a lixiviação. Em cada coluna, acondicionaram-se $350 \mathrm{~g}$ de solo, visando reproduzir a densidade encontrada no campo $\left(1.009,1.088,1.265\right.$ e $1.183 \mathrm{~kg} \mathrm{~m}^{-3}$ para o NV-A, NV-B, LV-A e LV-B, respectivamente). As colunas de solo foram lixiviadas com soluções de $\mathrm{HCl}$ 0,300 ou 1,650 mol L-1. Após a aplicação do ácido, o excesso de cloreto foi removido com água desionizada. A remoção do cloreto foi monitorada medindo-se a condutividade elétrica do líquido percolado. Para elevar o pH do solo, incubaram-se as amostras com $\mathrm{MgCO}_{3}$, de modo a fornecer 1,25 e 3,75 g de carbonatos por $\mathrm{kg}$ de solo para os tratamentos BASE1 e BASE2, respectivamente. As amostras desses solos foram incubadas por três meses antes de serem utilizadas nos estudos de adsorção. A faixa de umidade variou de $70 \%$ a $80 \%$ da umidade a $300 \mathrm{kPa}$ (Tabela 1). 
No experimento III, avaliou-se o efeito da adição de fosfatos e de sulfatos no solo. As amostras obtidas com a adição de fosfatos e sulfatos foram incubadas durante três meses antes de serem utilizadas nos estudos de adsorção, em condições análogas às do experimento II. Nos tratamentos com adição de fosfato (FOSF, ACIDFOSF e BASE-FOSF), adicionou-se $\mathrm{KH}_{2} \mathrm{PO}_{4}$, de modo a fornecer $300 \mathrm{mg}$ de $\mathrm{P}$ por $\mathrm{kg}$ de solo. Os tratamentos com aplicação de fosfato foram conduzidos estando o solo em diferentes CL denominados de FOSF, ACIDFOSF e BASE-FOSF, indicando que a adição de fosfatos foi feita ao LV-B, ACID2 e BASE2, respectivamente. No tratamento com aplicação de sulfato (SULF), incubou-se o solo com $\mathrm{CaSO}_{4} \cdot 2 \mathrm{H}_{2} \mathrm{O}$, de modo a fornecer $40 \mathrm{mg}$ de $\mathrm{S}$ por kg de solo. Este tratamento foi aplicado ao horizonte B do LV (LV-B). A discussão da influência dos diferentes tratamentos na adsorção de nitrato foi realizada com base nas alterações dos parâmetros de ajuste das isotermas de Freundlich, frente às alterações das cargas líquidas dos solos. O parâmetro $\mathrm{K}$ desse modelo permite identificar a capacidade de um solo em reter determinado soluto, possibilitando a estimativa da extensão do seu movimento na fase líquida. Este coeficiente é uma medida da distribuição de equilíbrio entre as fases sólida e líquida de um solo. Quanto maior a capacidade adsortiva, maior será o K. O parâmetro n indica a afinidade (energia de ligação) por sítios de troca e decresce exponencialmente com o aumento da adsorção (Bohn et al., 1985).

\section{Resultados e Discussão}

A CL dos solos variou entre $-99,2 \mathrm{e}+35,0 \mathrm{mmol}_{\mathrm{C}} \mathrm{kg}^{-1}$ e o $\mathrm{pH}$, de 3,70 a 7,24 para os dois solos e horizontes e nos diferentes tratamentos (Tabela 2). Em ambos os

Tabela 2. Cargas líquidas e pH dos solos nos tratamentos utilizados.

\begin{tabular}{llr}
\hline Tratamento & $\mathrm{pH}^{(1)}$ & $\mathrm{CL}^{(2)}$ \\
\hline NV-A & 5,27 & $-99,2$ \\
NV-B & 4,90 & $-31,6$ \\
LV-A & 4,46 & $-19,4$ \\
LV-B & 5,00 & $-3,2$ \\
ACID2 & 3,70 & $+35,0$ \\
ACID1 & 3,78 & $+34,0$ \\
BASE1 & 6,61 & $-19,0$ \\
BASE2 & 7,24 & $-24,0$ \\
FOSF & 5,60 & $-9,0$ \\
ACID-FOSF & 4,18 & $+30,0$ \\
BASE-FOSF & 7,11 & $-23,2$ \\
SULF & 5,49 & $-8,2$ \\
\hline
\end{tabular}

${ }^{(1)} \mathrm{pH}$ em KCl 1 mol L-1; relação solo:solução = 1:5. (2)Carga líquida $\left(\mathrm{mmol}_{\mathrm{c}} \mathrm{kg}^{-1}\right)$. solos, a CL foi algebricamente menor no horizonte superficial em relação ao subsuperficial, possivelmente pelo seu maior teor de matéria orgânica, uma fonte importante de cargas negativas no sistema (Parfitt, 1980; Bohn et al., 1985).

A carga superficial de um solo depende do seu pH, do $\mathrm{pH}_{0}$ (pH no qual a adsorção de $\mathrm{H}^{+}$é igual a de $\mathrm{OH}^{-}$), da concentração eletrolítica e da valência do contra-íon de sinal contrário (Bowden et al., 1980). Assim, considerando-se somente os tratamentos aplicados no horizonte B do $\mathrm{LV}$, houve uma correlação significativa entre a CL e o pH do solo $\left(\mathrm{R}^{2}=0,917^{* *}\right)$ (Figura 1). Quando se consideram todos os tratamentos, essa correlação deixa de ser significativa $\left(\mathrm{R}^{2}=0,226^{\mathrm{ns}}\right)$. Os diversos constituintes dos solos que contribuem para a formação de carga apresentam comportamento diferenciado frente às alterações de $\mathrm{pH}$. Assim, na presença de amostras com composição química e mineralógica distintas, a relação entre a carga do solo e a variação do $\mathrm{pH}$ do meio fica mascarada (Gillman, 1979).

Com a adição de carbonato de magnésio nos tratamentos BASE1 e BASE2, a CL das amostras diminuiu algebricamente de -3,2 para -19,0 e -24,0 mmol $_{\mathrm{C}} \mathrm{kg}^{-1}$ de solo. A adição de ácido (ACID1 e ACID2) aumentou a CL do horizonte B do LV de $-3,2$ para $+34,0$ e $+35,0 \mathrm{mmol}_{\mathrm{C}} \mathrm{kg}^{-1}$ de solo. Embora tenha sido grande a concentração de ácido utilizada nos tratamentos ACID1 e ACID2, a variação na CL foi pequena. Do mesmo modo, o pH original das amostras sofreu alteração de 5,0 para 3,78 e 3,70 em decorrência dessa adição. A adição de sulfato (SULF) resultou em pequeno au-

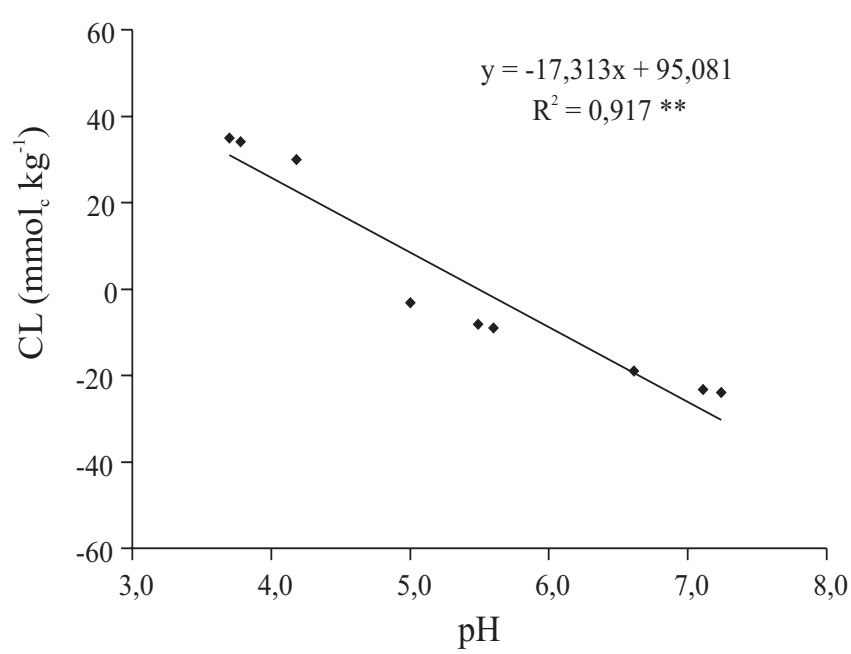

Figura 1. Relação entre o pH e a carga líquida (CL) obtida para os tratamentos utilizados no horizonte B do Latossolo Vermelho acriférrico. ${ }^{* *}$ Significativo a $1 \%$ de probabilidade pelo teste de Tukey. 
mento no pH do horizonte B do LV, que passou de 5,00 para 5,49. Por sua vez, a CL foi algebricamente reduzida de $-3,2$ para $-8,2 \mathrm{mmol}_{\mathrm{C}} \mathrm{kg}^{-1}$. A adição de fosfato (FOSF) teve um efeito levemente mais pronunciado: o $\mathrm{pH}$ e a CL foram modificados de 5,00 para 5,60 e de -3,2 para -9,0 $\mathrm{mmol}_{\mathrm{C}} \mathrm{kg}^{-1}$, respectivamente.

Existem limites para a variação do pH e da CL de um solo. A alteração do pH do solo pela adição de ácido ou de base não é sempre linear. Como a curva de $\mathrm{pH}$ em função da adição de ácidos e de bases tem um comportamento seno-hiperbólico, à medida que se afasta do PESN do solo (extremidades da curva), adições de quantidades iguais de ácido ou de base irão resultar em variações cada vez menores de $\mathrm{pH}$ do solo. $\mathrm{O}$ controle dessa variação, tanto na parte ácida como na básica, é definido pelo desmonte da rede cristalina, o que ocorre em torno de pH 2,5 a 3,0 e de 9,0 a 9,5 (Uehara \& Gillman, 1981). A variação de pH obtida não extrapolou esses limites.

Os coeficientes de determinação $\left(\mathrm{R}^{2}\right)$ variaram de 0,912 a 0,996 , sendo todos significativos a $1 \%$ de probabilidade pelo teste de Tukey, indicando que o modelo de Freundlich conseguiu descrever satisfatoriamente a adsorção do nitrato aos solos (Tabela 3). Em nenhum dos tratamentos foi possível ajustar o modelo de Langmuir aos dados obtidos com o programa computacional utilizado. Black \& Waring (1976) também observaram que as isotermas de adsorção de nitrato se ajustaram à equação de Freundlich, mas não à de Langmuir. Conforme Bohn et al. (1985), a equação de Freundlich é a que melhor descreve os dados das concentrações adsorvidas quando a máxima adsorção não é determinada.

Tabela 3. Parâmetros de ajuste das isotermas de Freundlich aos solos utilizados.

\begin{tabular}{lccc}
\hline Tratamento & $\mathrm{K}^{(1)}$ & $\mathrm{n}^{(2)}$ & $\mathrm{R}^{2}$ \\
\hline NV-A & $0,115 \pm 0,07$ & $4,195 \pm 0,05$ & $0,976^{* *}$ \\
NV-B & $0,071 \pm 0,07$ & $4,383 \pm 0,77$ & $0,943^{* *}$ \\
LV-A & $0,358 \pm 0,18$ & $3,710 \pm 0,46$ & $0,974^{* *}$ \\
LV-B & $0,689 \pm 0,46$ & $3,066 \pm 0,59$ & $0,912^{* *}$ \\
ACID2 & $8,073 \pm 0,62$ & $0,709 \pm 0,10$ & $0,946^{* *}$ \\
ACID1 & $0,827 \pm 0,36$ & $2,913 \pm 0,47$ & $0,982^{* *}$ \\
BASE1 & $6,653 \pm 0,48$ & $0,800 \pm 0,09$ & $0,923^{* *}$ \\
BASE2 & $4,176 \pm 0,48$ & $1,403 \pm 0,15$ & $0,994^{* *}$ \\
FOSF & $0,263 \pm 0,08$ & $3,626 \pm 0,28$ & $0,982^{* *}$ \\
ACID-FOSF & $1,134 \pm 0,20$ & $2,550 \pm 0,19$ & $0,996^{* *}$ \\
BASE-FOSF & $0,541 \pm 0,09$ & $3,067 \pm 0,16$ & $0,991^{* *}$ \\
SULF & $0,388 \pm 0,13$ & $3,291 \pm 0,32$ & $0,994^{* *}$ \\
\hline
\end{tabular}

${ }^{(1)}$ Coeficiente de adsorção $\left(10^{-6} \mathrm{~mol}^{(1-n)} \mathrm{m}^{3(\mathrm{n}-1)}\right)$. (2)Estimativa do parâmetro de ajuste (adimensional). ${ }^{* *}$ Significativo a $1 \%$ de probabilidade pelo teste de Tukey.
Nos tratamentos LV-A e LV-B, os valores de K foram 0,358 e $0,689 \times 10^{-6} \mathrm{~mol}^{(1-n)} \mathrm{m}^{3(\mathrm{n}-1)}$, respectivamente. Para o NV, o K foi igual a 0,115 e $0,071 \times 10^{-6} \mathrm{~mol}^{(1-n)} \mathrm{m}^{3(\mathrm{n}-1)} \mathrm{nos}$ horizontes superficial e subsuperficial, respectivamente (Tabela 3). Tais resultados indicam a maior capacidade adsortiva do LV em relação ao NV. Estes valores podem ser explicados pelas diferenças nas CL entre os solos, ou seja, a CL do LV foi algebricamente maior (menos negativa) do que a do $\mathrm{NV}$, o que favoreceria a maior adsorção de nitrato naquele solo (Tabela 2). No NV, os valores de $\mathrm{n}$ foram $4,195 \times 10^{-6} \mathrm{~mol}^{(1-n)} \mathrm{m}^{3(\mathrm{n}-1)}$ e $4,383 \times 10^{-6} \mathrm{~mol}^{(1-n)} \mathrm{m}^{3(\mathrm{n}-1)}$ nos horizontes A e B, respectivamente. No LV, esses valores foram $3,710 \times 10^{-6} \mathrm{~mol}^{(1-n)} \mathrm{m}^{3(\mathrm{n}-1)}$ e $3,066 \times 10^{-6} \mathrm{~mol}^{(1-\mathrm{n})} \mathrm{m}^{3(\mathrm{n}-1)}$. Os menores valores de $\mathrm{n}$ no LV em relação ao NV indicam maior afinidade do nitrato pelos sítios de adsorção naquele solo, corroborando as discussões em relação ao parâmetro $\mathrm{K}$ (Figura 2).

Ao se comparar a adsorção de nitrato nos horizontes A e B de um mesmo solo, o efeito não é tão evidente em relação ao observado, considerando-se diferentes solos. No caso do NV, o parâmetro n foi pouco maior no horizonte superficial em relação ao subsuperficial (4,195 e 4,383, respectivamente), indicando maior capacidade adsortiva do horizonte A em relação ao B. O mesmo aconteceu com o $\mathrm{K}\left(0,115 \times 10^{-6} \mathrm{~mol}^{(1-\mathrm{n})} \mathrm{m}^{3(\mathrm{n}-1)}\right.$ e $0,071 \times 10^{-6} \mathrm{~mol}^{(1-\mathrm{n})} \mathrm{m}^{3(\mathrm{n}-1)}$ nos horizontes Ae B, respectivamente). Nesse caso, as diferenças na adsorção não podem ser explicadas somente pela alteração na CL.

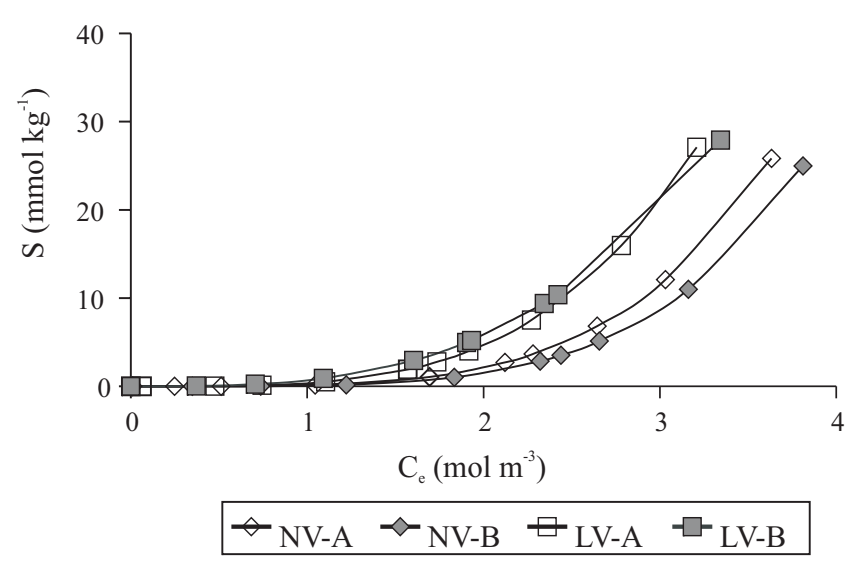

Figura 2. Efeito dos solos na adsorção de nitrato. S: nitrato adsorvido ao solo (valores ajustados); $\mathrm{C}_{\mathrm{e}}$ : nitrato na solução após a agitação. 
No NV, a CL foi menor algebricamente no horizonte A em relação ao B (-99,2 e -31,6 mol $\left._{\mathrm{C}} \mathrm{m}^{-3}\right)$, indicando que a adsorção de nitrato seria maior no horizonte $B$. No entanto, é preciso lembrar que a carga líquida é um balanço entre as cargas positivas e negativas do solo, que coexistem. Assim, a capacidade de troca de ânions no horizonte A desse solo é levemente maior do que a encontrada no horizonte $B\left(5,1 \mathrm{~mol}_{\mathrm{C}} \mathrm{m}^{-3} \mathrm{e} 4,8 \mathrm{~mol}_{\mathrm{C}} \mathrm{m}^{-3}\right)$ (Tabela 1), o que justificaria a maior afinidade no horizonte superficial, uma vez que a CTA é uma medida das cargas positivas do solo.

As curvas praticamente coincidentes entre os tratamentos ACID1 e LV-B indicam que o nível menor de acidificação não alterou a adsorção de nitrato em relação ao observado em condições naturais (Figura 3). Por sua vez, o tratamento ACID2 aumentou a inclinação da isoterma em relação ao LV-B, indicando que o equilíbrio entre as fases adsorvidas e em solução foi deslocado em direção à fase sólida. Esse deslocamento no equilíbrio é confirmado pelas alterações nos parâmetros de ajuste das isotermas de adsorção (Tabela 3). No tratamento ACID2, K aumentou de $0,689 \times 10^{-6} \mathrm{~mol}^{(1-n)} \mathrm{m}^{3(\mathrm{n}-1)}$ para $8,074 \times 10^{-6} \mathrm{~mol}^{(1-n)} \mathrm{m}^{3(\mathrm{n}-1)}$. A acidificação também aumentou a afinidade pelos sítios de adorção: o n passou de 3,066 em condições naturais para 0,709. Kinjo \& Pratt (1971) constataram relação entre diminuição do pH na suspensão até 3,5 e aumento na adsorção de nitrato. Os resultados podem ser ao menos parcialmente explicados pela inversão da CL originalmente presente no solo em conseqüência da acidificação, o que favoreceu a adsorção do ânion nos sítios de troca do solo (Tabela 2).

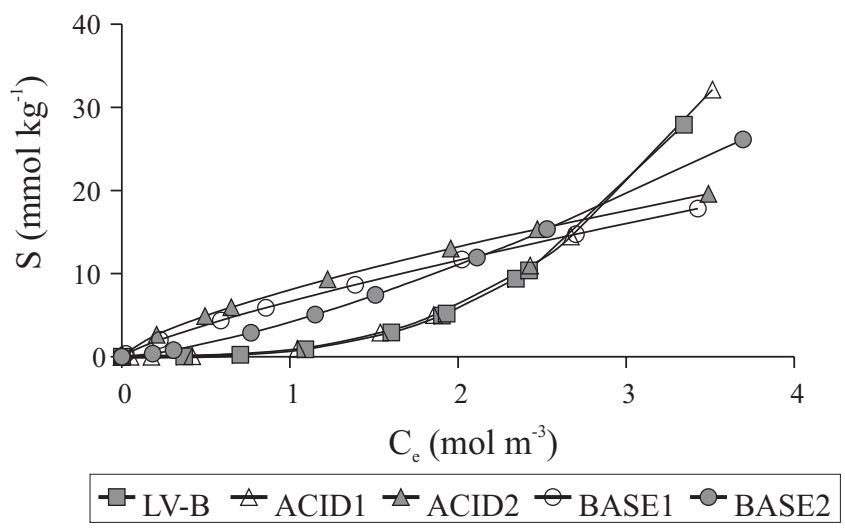

Figura 3. Efeito da adição de ácidos e de bases na adsorção de nitrato. S: nitrato adsorvido ao solo (valores ajustados); $\mathrm{C}_{\mathrm{e}}$ : nitrato na solução após a agitação.
Como a elevação do pH do solo resultou em diminuição algébrica da CL, esperava-se que a adsorção de ânions fosse menor do que a observada em condições de $\mathrm{pH}$ natural (Figura 3). Porém, as isotermas de adsorção mostram o inverso (o aumento do $\mathrm{pH}$ favoreceu a adsorção), o que é corroborado pelos parâmetros de ajuste das isotermas. Nos tratamentos BASE1 e BASE2 o n foi de 0,800 e 1,403, respectivamente. Esses valores são menores do que o $\mathrm{n}$ ajustado para o LV-B, indicando maior afinidade pelos sítios de troca do solo. Também o comportamento do parâmetro $\mathrm{K}$ nos tratamentos BASE1 e BASE2 foi inesperado, aumentando para $6,653 \times 10^{-6} \mathrm{~mol}^{(1-n)} \mathrm{m}^{3(\mathrm{n}-1)}$ e $4,176 \times 10^{-6} \mathrm{~mol}^{(1-\mathrm{n})} \mathrm{m}^{3(\mathrm{n}-1)}$, respectivamente.

A adição de sulfato ao solo resultou em pequena diminuição na adsorção de nitrato em relação à observada no LV-B (Figura 4). Esse comportamento é corroborado pelas alterações nos parâmetros de ajuste das isotermas (Tabela 3). O parâmetro $\mathrm{n}$ da equação de Freundlich obtido para o LV-B aumentou de 3,066 para 3,291 no tratamento SULF. Também o parâmetro $\mathrm{K}$ foi reduzido de $0,689 \times 10^{-6} \mathrm{~mol}^{(1-\mathrm{n})} \mathrm{m}^{3(\mathrm{n}-1)}$ para $0,388 \times 10^{-6} \mathrm{~mol}^{(1-\mathrm{n})} \mathrm{m}^{3(\mathrm{n}-1)}$. Tais resultados podem ser explicados, ao menos parcialmente, pela diminuição algébrica da CL do solo provocada pela adição de sulfato, que passou de -3,2 para -8,2 $\mathrm{mmol}_{\mathrm{C}} \mathrm{kg}^{-1}$ (Tabela 2). A menor adsorção de nitrato com a adição de sulfato ao solo já foi detectada por Kinjo \& Pratt (1971), trabalhando em solos ácidos. Oliveira et al. (2000), ao aplicarem gesso agrícola $\left(\mathrm{CaSO}_{4} \cdot 2 \mathrm{H}_{2} \mathrm{O}\right)$ em subsolos de Cerrado com carga variável, também obtiveram redução no parâmetro n. O sulfato, por ser um oxiânion, com-

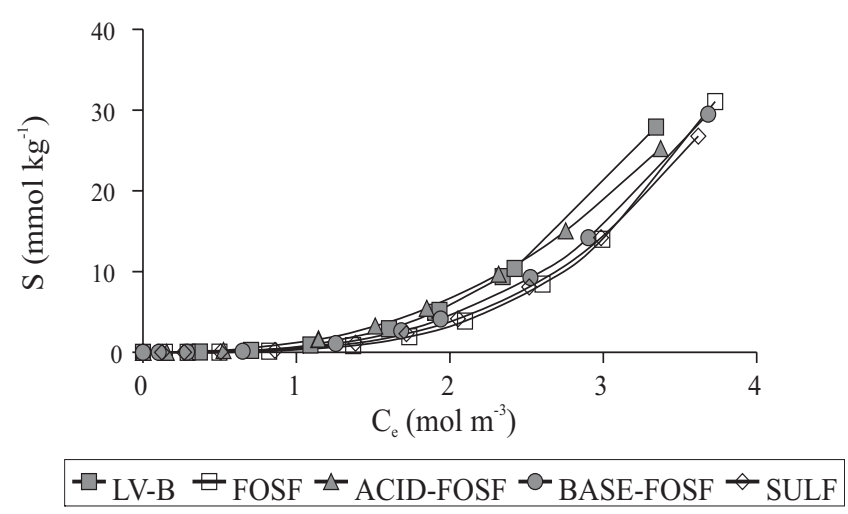

Figura 4. Efeito da adição de sulfato e de fosfato na adsorção de nitrato. S: nitrato adsorvido ao solo (valores ajustados); $\mathrm{C}_{\mathrm{e}}$ : nitrato na solução após a agitação. 
petiria com o nitrato pelos sítios de troca carregados positivamente, o que resultaria em menor adsorção de nitrato ao solo.

A adição de fosfato ao solo também resultou em pequena redução na adsorção de nitrato em relação ao LV-B (Figura 4). A isoterma de adsorção no tratamento FOSF apresenta-se levemente deslocada para a direita em relação ao LV-B, indicando que o equilíbrio entre a fase adsorvida e a fase em solução foi deslocado para a fase líquida. Os parâmetros calculados no ajuste da equação de Freundlich corroboram esse comportamento. Com a adição de fosfato, o $\mathrm{n}$ aumentou de 3,066 para 3,626, ao passo que o $\mathrm{K}$ diminuiu de $0,689 \times 10^{-6} \mathrm{~mol}^{(1-\mathrm{n})} \mathrm{m}^{3(\mathrm{n}-1)}$ para $0,263 \times 10^{-6} \mathrm{~mol}^{(1-\mathrm{n})} \mathrm{m}^{3(\mathrm{n}-1)}$. Da mesma forma, isso também ocorreu quando a adição de fosfato foi feita ao solo que recebeu adição de ácido (ACID-FOSF) ou de base (BASE-FOSF). Em experimento no campo em solo de Cerrado, Dynia \& Camargo $(1998,1999)$ verificaram o efeito da aplicação de calcário ou de calcário mais fosfato na capacidade de retenção de nitrato do solo (CRN). Os autores encontraram relação entre a diminuição da CRN e as alterações eletroquímicas (CTC, CTA e CL) provocadas pelos tratamentos aplicados. Essa relação depende do tipo e da quantidade de cargas variáveis e permanentes dos minerais presentes no solo e da composição dos complexos de troca. $\mathrm{O} \mathrm{SO}_{4}{ }^{2-}$ e o $\mathrm{H}_{2} \mathrm{PO}_{4}{ }^{2-}$ conseguem ocupar os mesmos sítios de adsorção ocupados pelo $\mathrm{NO}_{3}{ }^{-}$, mas suas afinidades seriam diferentes. Como o ânion $\mathrm{H}_{2} \mathrm{PO}_{4}{ }^{2-}$ apresenta maior afinidade pelos sítios de troca em relação ao $\mathrm{NO}_{3}{ }^{-}$, ele os preencheria previamente. O preenchimento dos sítios de troca por fosfato ou nitrato não é uma questão tão simples, uma vez que dois ou mais sítios de adsorção podem estar envolvidos na adsorção do fosfato (Kinjo \& Pratt, 1971). Além disso, a adsorção de fosfato não é somente eletrostática, mas significativamente específica.

\section{Conclusões}

1. Há correlação significativa entre o pH e a carga líquida nas amostras do horizonte B do Latossolo Vermelho acriférrico nos diversos tratamentos.

2. O modelo de Freundlich descreve satisfatoriamente a adsorção do nitrato ao solo.

3. A adsorção do nitrato nos horizontes A e B do Latossolo Vermelho acriférrico é maior no horizonte subsuperficial em relação ao superficial e no Nitossolo Vermelho eutrófico, é maior no horizonte superficial em relação ao subsuperficial.
4. A adsorção de nitrato acompanha a variação da carga líquida obtida, com exceção nos tratamentos que recebem adição de bases.

\section{Agradecimentos}

Ao CNPq, pela bolsa concedida a Otávio Antonio de Camargo e pelo apoio financeiro; à Fapesp, pelo apoio financeiro; ao Dr. Ricardo Marques Coelho, Pesquisador do IAC/CPDSRA, pela na revisão do artigo.

\section{Referências}

BAKER, A.S. Extracting solution for potentiometric determination of nitrate in plant tissue. Journal of Agricultural and Food Chemistry, v.17, p.802, 1969.

BELLINI, G.; SUMNER, M.E.; RADCLIFFE, D.E.; QAFOKU, N.P. Anion transport through columns of highly weathered acid soils: adsorption and retardion. Soil Science Society of American Journal, v.60, p.132-137, 1996.

BLACK, A.S.; WARING, S.A. Nitrate leaching and adsorption in a Krasnozem from Redland Bay, Qld III. Effect of nitrate concentration on adsorption and movement in soil columns. Australian Journal of Soil Research, v.14, p.189-195, 1976.

BOHN, H.L.; McNEAL, B.L.; O’CONNOR, G.A. Soil Chemistry. New York: John Wiley, 1985. 341p.

BOWDEN, J.W.; POSNER, A.M.; QUIRK, J.P. Adsorption and charging phenomena in variable charge soils. In: THENG, B.K.G. (Ed.). Soils with variable charge. Palmerston North, New Zealand: New Zealand Society of Soil Science, 1980. p.147-166.

CAMARGO, O.A. de; RAIJ, B. van. Movimento do gesso em amostras de latossolos com diferentes propriedades eletroquímicas. Revista Brasileira de Ciência do Solo, v.13, p.275-280, 1989.

CAMARGO, O.A. de; MONIZ, A.C.; JORGE, J.A.; VALADARES, J.M.A.S. Métodos de análise química, mineralógica e física de solos do Instituto Agronômico de Campinas. Campinas: Instituto Agronômico, 1986. 94p. (Boletim técnico, 106).

DYNIA, J.F.; CAMARGO, O.A. de. Effects of liming, green manure, and phosphate addition on electrochemical attributes of an oxisol from Central Brazil. Pesquisa Agropecuária Brasileira, v.29, p.755762, 1998.

DYNIA, J.F.; CAMARGO, O.A. de. Nitrate retention in a variablecharge soil, as influenced by phosphate fertilizing and liming. Pesquisa Agropecuária Brasileira, v.34, p.141-144, 1999.

EMBRAPA. Centro Nacional de Pesquisa de Solos (Rio de Janeiro, RJ). Sistema brasileiro de classificação de solos. Brasília: Embrapa-SPI; Rio de Janeiro: Embrapa-CNPS, 1999. 412p.

GILLMAN, G.P. A proposed method for the measurement of exchange properties of highly weathered soils. Australian Journal of Soil Research, v.17, p.129-139, 1979.

KINJO, T.; PRATT, P.F. Nitrate adsorption: 1. In some acid soils of Mexico and South America. Soil Science Society of America Proceedings, v.35, p.722-725, 1971. 
NAIDU, R.; SYERS, J.K.; TILLMAN, R.W.; KIRKMAN, J.H. Effect of liming and added phosphate on charge characteristics of acid soils. Journal of Soil Science, v.41, p.157-164, 1990.

OLIVEIRA, J.R.A. de; VILELA, L.; AYARZA, M.A. Adsorção de nitrato em solos de Cerrado do Distrito Federal. Pesquisa Agropecuária Brasileira, v.35, p.1199-1205, 2000.

PARFITT, R.L. Chemical properties of variable charge soils. In: THENG, B.W.K. (Ed.). Soils with variable charge. Palmerston North, New Zealand: Society of Soil Science, 1980. p.167-194.

RAIJ, B. van; CAMARGO, O.A. de. Nitrate elution from soil colunms on three Oxisols and one Alfisol. In: INTERNATIONAL
CONGRESS OF SOIL SCIENCE, 1., Moscou, 1974. Transaction of the $\mathbf{1 0}^{\text {th }}$ International Congress of soil Science. v.2, p.384391.

SELIM, H.M.; AMACHER, M.C. Reactivity and transport of heavy metals in soils. New York: Boca Raton, 1997. 201p.

UEHARA, G.; GILLMAN, E. The mineralogy, chemistry, and physics of tropical soils with variable charge clays. Bauder: Westview Press, 1981. 170p. (Westview Tropical Agriculture, 4).

WONG, M.T.F.; VANDERKRUIJS, A.C.B.M.; JUO, A.S.R. Leaching loss of calcium, magnesium, potassium and nitrate derived from soil, lime and fertilizers as influenced by urea applied to undisturbed lysimeters in south-east Nigeria. Fertilizer Research, v.31, p.281-289, 1992.

Recebido em 19 de maio de 2004 e aprovado em 25 de janeiro de 2005 